\title{
Research on Smart Ocean Engineering Construction
}

\author{
Haitao $\mathrm{Li}^{1, \text { a) }}$, Lei Zhang ${ }^{1, \mathrm{~b})}$ and Haiguang Huang ${ }^{23, \mathrm{c})}$ \\ ${ }^{1}$ College of Information Science and Technology, Qingdao University of Science and Technology, \\ Qingdao 266061, China. \\ ${ }^{2}$ College of Information Science and Engineering, Ocean University of China, Qingdao 266100, \\ China;
}

${ }^{3}$ Wenzhou ocean and fishery vessel safety rescue information center, Wenzhou

\author{
a) 32891741@qq.com \\ b)1336482253@qq.com \\ c) haiguang2000@gmail.com
}

Keywords: Smart, Ocean, Construction

\begin{abstract}
At present, the construction of ocean information has become an important support for China's ocean power strategy, but the lack of unified planning in the process of construction, homogeneity serious, information island, wisdom is not enough and other issues become increasingly obvious, this article from the integrated planning and construction ideas, this paper puts forward a scheme of smart ocean engineering construction based on human body theory, which is constructed from the three-dimensional monitoring system of ocean environment, and the ocean Large-scale data platform construction, ocean application system development to information standards and norms, and other aspects of a complete program design, the program covers the ocean environmental protection, disaster prevention and mitigation, sea islands, ocean law enforcement, ocean economy, ocean fisheries and other business category. The research results are practiced in the construction of the wisdom of the coastal provinces of China, and the wisdom of the other coastal provinces, the wisdom of the sea has a greater reference.
\end{abstract}

\section{INTRODUCTION}

In order to speed up the construction of Chinese modern ocean and fishery industry system, improve the comprehensive strength of the ocean and fishery economy and realize the strategic goal of the ocean power, Chinese coastal economically developed provinces have implemented a series of ocean information construction, but there still exists a series of problem such as lack of unified programming, homogeneous serious, information isolated-islands and not enough wisdom. Therefore, this paper puts forward the idea of smart ocean engineering construction based on human body theory. It aims to achieve a comprehensive stereo perception of the ocean, extensive connectivity, share of massive data, scientific management and analysis, smart decision-making and service with the help of human biological theory.

\section{PROGRAM OF SMART OCEAN ENGINEERING CONSTRUCTION}

The monitoring equipment is similar to huam facial feature, which build a comprehensive perception of monitoring and management thoughout reasonable layout and division of work. Similar to human skeleton, the PC, server, storage devices, room, conference room and other hardware infrastructure are the support of smart ocean. Similar to neutal network of the human body, the network architecture of the smart ocean is complicated. However, in the overall planning and design, it should effectively integrate and subdivide each vein so that they can carry out their duties but also achieve high degree of unity. Similar to the function of human brain, the wisdom decisionmaking of smart ocean conduct ,coordinate and track the body function. Seas and islands, resources and environment, forecasting and disaster reduction, fishery synthesis, ocean law enforcement and 
administrative integrated management system is like limbs of human body, which have clear responsibilities and coordinate to work forward. Big data of the smart ocean is like cells in human body, which are large volume. So when we design this, we should construct operating mechanism of the system, which can collect data, manage data, mainten data and update data.

\section{Stero Monitoring Network of Smart Ocean}

The development and application of big data cannot separate from support of data. So we should construct stereo monitoring network of smart oean in order to achieve collecting equipment data, and monitoring network data transmission. At present, Chinese coastal provinces and cities are already or preliminarily provided with their own monitoring equipment and network. The monitoring equipment includes buoy, submersible, ground wave radar, seabed base, survey section, subocean observation network, video surveillance, satellite remote sensing, forecasting ship, unmanned aerial vehicle, underwater glider, multi-beam, ROV robot, offshore platform, ADCP ,the sidetracking, and etc. The monitoring network includes wireless network, satellite network, Internet, ocean green network, ocean and fishery government network, national sea network, e-government network and so on. Based on theae equipment, we should further expand internet of things, internet, video surveillance network and other terminal equipment deployment so as to enhance the awareness ability of data terminal. At the same time, this can achieve network integration,and interoperability so as to facilitate the comprehensive analysis and deep mining of data and further provide a steady stream of data support.

\section{Big Data Platform of Smart Ocean}

Generally speaking, big data should be large and complex. In other words, it is data set which content cannot be crawled, managed and handled with traditional database software tools. It has the following four characteristics(4V) [7]:

(1) Volumes: large data sets, jump from TB level to PB and even higher level.

(2) Variety: data derives from various data sources ,data types and formats break through the previously defined structured data categories including audio and video files and other semistructured and unstructured data.

(3) Value: low data value density

(4) Velocity: data processing speed requires fast, it needs online analysis and real-time data processing

Compared with the above characteristics, with the construction and improvement of ocean stereo monitoring network, ocean data show explosive growth phenomenon. Coupled with its characteristics of multi-source and heterogeneous, it is a new challenge for ocean management departments and fans of the ocean business units even individuals to effectively manage, mainten, share, analysis and mine, ocean big data. As shown in the figure 3, ocean big data sources can be categorized into six classes including ocean monitoring and evaluation, ocean observation and forecasting, management of seas and islands, ocean administrative law enforcement, ocean economy and ocean fishery. Each category can form independent system but interrelated. Therefore, in the process of construction of ocean big data platform, we should fully consider utilize and mine data from all aspects as well as generated value by overall integration and analysis.

\section{CONSTRUCTION OF OCEAN BIG DATA PLATFORM}

(1) Framework of big data platform

Based on the above six classes of ocean big data, we construct ocean big data platform as figure 4. Ocean big data platform include six levels: data integration layer, data storage management layer, programming model layer, data analysis layer, visual data product layer and decision-making and publishing layer.

Data integration layer: this platform provides rich data integration interface to support the traditional relational database products and Internet, Internet of Things application data collection interface seamless integration.So it can easy to import multi-source heterogeneous data into the platform data storage system.

Data Cloud Storage Management: it provides PB-level structured and unstructured data cloud 
storage and management so as to support basic data set operations such as efficient data query, data index and data extraction.

Programming model layer: it provide WEB-based and workflow-based data mining model support. It is easy for analysis staff to online edite and submit analysis model anytime and anywhere.

Data analysis layer: it provide Hadoop-based offline analysis environment and Spark-based online analysis environment in order to meet needs of different application scenarios for data analysis response efficiency. Data analysis techniques include classification, clustering, association rules, genetic algorithms, neural networks, predictive models, pattern recognition, time series analysis, regression analysis, system simulation, machine learning, optimization, spatial analysis, and social network analysis. Through the real-time data analysis and processing, it can be used in ocean disaster prevention and mitigation, ocean environmental monitoring, ocean fishery forecast and other fields [5]

Visual data product layer: it can provide visual analysis and report support.Users can carry out interactive visual data products manufacture through visual analysis tools, visualization engine, report templates and other functions. It can give an dynamic,visual and multi-dimensional expression for ocean elements, ocean processes, ocean forecasting through the establishment of ocean data analysis and application model [5]. Thus, it will provide information visualization services for ocean scientific research and industry applications.

Decision-making and publishing layer: Based on the above platform system, through the web site, WeChat public number, mobile App, microblogging, fax, it provide the ocean field related units and individuals fast real-time decision support data services, including forecasting, ocean pollution monitoring and alarm, ocean fishery monitoring, ocean main rights command and so on.

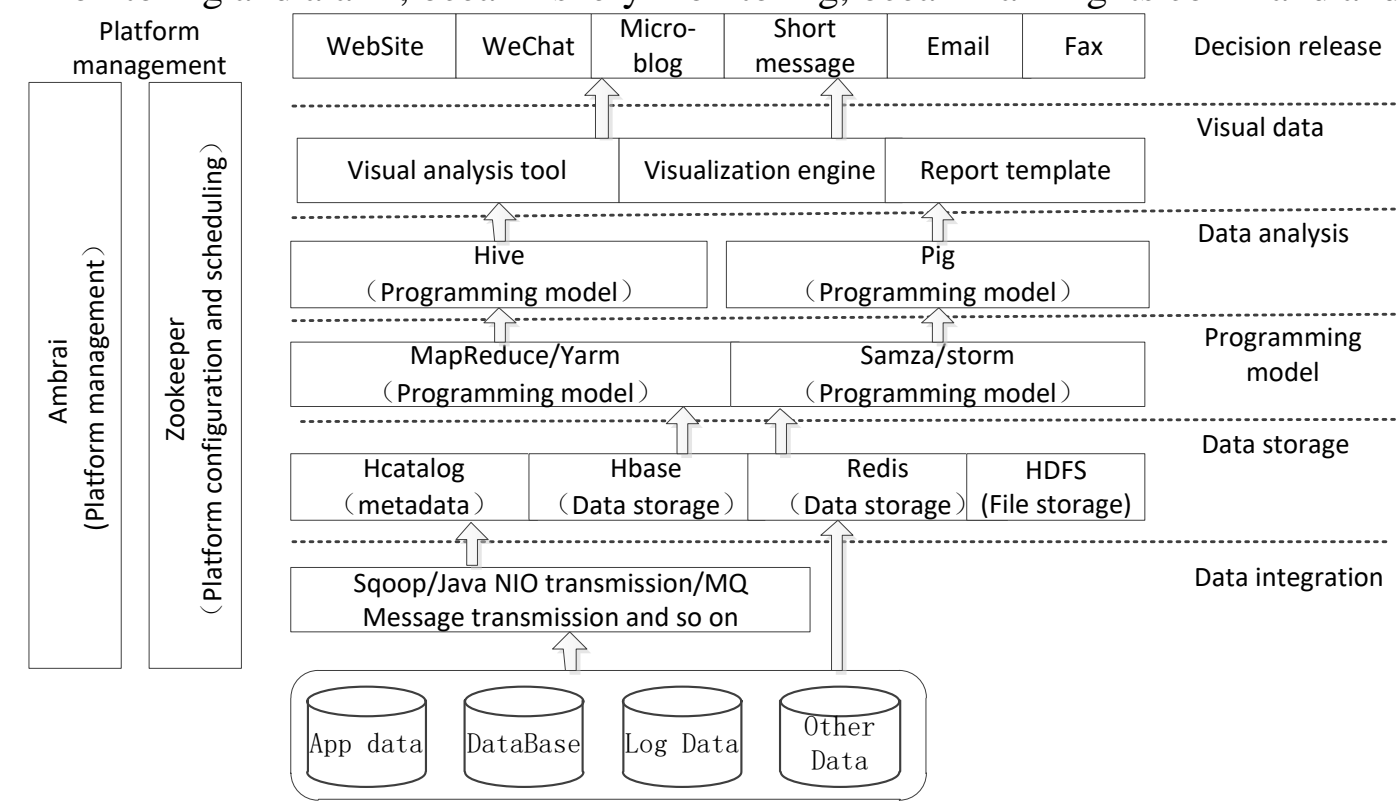

Fig 4 Big data platform Framework

\section{Integrated Management Platform for Smart Ocean}

On the basis of unified standards and frameworks, it is essential to build integrated management platform of smart ocean for department of ocean and fishery government management, ocean fishery enterprises and the public. This platform should cover Ocean ecological and environmental protection, Ocean forecast and disaster mitigation, sea island management, Ocean administrative law enforcement, Ocean economy, Ocean fisheries, and other fields. It is a key point to build urgently demand and remarkable effect project such as the ocean environment monitoring and evaluation system, ocean environmental wisdom forecasting system, the wisdom fishery cloud picture management system service platform, ocean resources, ocean emergency management system. 


\section{APPLICATION SYSTEM OF SMART OCEAN}

(1) Ocean ecological environmental protection

Construct ocean environment three-dimensional real-time monitoring system, smart ocean environmental monitoring and evaluation system, ocean resources, smart management of a map system, ocean protection and development of information systems. Through Through the construction of smart ocean environmental monitoring, evaluation, management, decision-making and other service systems, it can strengthen the construction of ocean ecological civilization, comprehend the transformation of ocean economic development and industrial structure optimization.

(2) Ocean forecasting and disaster mitigation

Construct ocean disaster environment real-time monitoring system, ocean environmental intelligence forecasting and early warning system, ocean pre-alarm comprehensive production and distribution system, ocean emergency emergency management system.

(3) Management of seas and islands

Construct dynamic monitoring system for seas and islands, management system for sea use, smart analysis and decision system for seas and islands, dynamic information publication system for seas and islands, control information system for the ocean functional area, three-dimentional visualization system for island.

(4) Ocean administrative law enforcement

Construct ocean law enforcement supervision information management system, ocean law enforcement monitoring smart auxiliary decision-making system, ocean law enforcement supervision information release system, ocean rights and interests maintenance information management system.

(5) Ocean economy

Construct ocean economic operation monitoring and evaluation system, ocean economic geographic information system, ocean economic information sharing system, ocean economic public opinion monitoring system.

(6) Ocean fishery

Construct fishery environment smart monitoring and evaluation system, aquaculture intelligence decision support system, aquaculture smart remote diagnosis system, aquatic product quality and safety management system, fishery integrated fishing management system, fishery production safety management system, fishing port management system.

\section{CONCLUSION}

Based on the human body theory of smart ocean engineering construction program provides the maine data perception and transmission, mass data storage and calculation, integrated management of ocean business and smart evaluation and decision-making integration of the planning and construction programs. It provide for the owners effective and smart information. The construction of ideas, respectively be implement in these process of smart ocean construction such as Smart Ocean of Guangxi, Smart Ocean of Wenzhou, Smart Ocean of Huizhou and so on.

\section{REFERENCES}

[1].Xing Yun. "13th Five-Year" planning. Ocean satellite application, 201606.

[2].Hongtao Zhang. "The development of smart ocean power construction" wisdom ocean and IOT Development Summit Forum "held". China ship report, 2015-06-12.

[3]."The Central Committee of the Communist Party of China on the formulation of national economic and social development of the thirteenth year plan proposal", 2015-10-29.

[4].Zhihui Wang Internet plus ocean = "Smart ocean. China ship report, 2015-07-17.

[5].Hong Yang, Hou Jian. The construction and application of ocean big data platform. Satellite application, the 201 phase of the year 06. 
[6].Ahlswede R, Cai N, Li S Y R et al.Network Information Flow J.IEEE Trans.on Inform.Theory, 2000, 46 (1): 12041216. 